\title{
Acute Spontaneous Cervical Epidural Hematoma Mimicking Cerebral Stroke: A Case Report and Literature Review
}

\author{
Jin Kyu Kim, Tae Hong Kim, Sang Keun Park, Yong Soon Hwang, \\ Hyung Shik Shin, Jun Jae Shin \\ Department of Neurosurgery, Sanggye-Paik Hospital, Inje University College of Medicine, Seoul, Korea
}

Spontaneous cervical epidural hematoma (SCEDH) is a rare disease, but can cause severe neurologic impairment. We report a case of a 68-year-old female who presented with sudden onset, posterior neck pain, right shoulder pain, and progressive right hemiparesis mimicking stroke with no trauma history. Initial brain CT and diffusion MRI performed to rule out brain lesion did not show any positive findings. Laboratory examination presented only severe thrombocytopenia $\left(45,000 / \mathrm{mm}^{3}\right)$. Subsequent cervical MRI revealed a cervical epidural mass lesion. We confirmed that it was pure hematoma through C5 unilateral total laminectomy and C6 partial hemilaminectomy. She achieved complete neurologic recovery with active rehabilitation. Early surgical decompression for SCEDH with neurologic impairment should be recommended for better outcome.

Key Words: Spontaneous cervical epidural hematoma $(\mathrm{SCEDH}) \cdot$ Stroke $\cdot$ Liver cirrhosis

\section{INTRODUCTION}

Spontaneous cervical epidural hematoma (SCEDH) is an uncommon disorder and can develop signs and symptoms of spinal cord compression. Clinically, it can present as a wide range of neurological deficits from simple cervical radiculopathy to complete quadriplegia depending on the severity and rapidity of compression. Such neurological deficits are reported to be associated with blood coagulopathies, anti-coagulant treatment, infection, tumor genesis, pregnancy, herniated discs, Paget's disease, and vascular malformations ${ }^{8)}$. Although several predisposing factors have been proposed, the exact bleeding source and mechanism are still uncertain. Recently, we successfully treated a case of spontaneous cervical epidural hematoma mimicking epidural mass-like lesion with different MRI findings in a liver cirrhosis patient.

\section{CASE REPORT}

A 68-year-old female presented with sudden onset posteri-

- Received: April 27, 2013 • Revised: August 25, 2013

- Accepted: August 27, 2013

Corresponding Author: Tae Hong Kim, MD

Department of Neurosurgery, Sanggye-Paik Hospital, Inje University College of Medicine, Seoul 139-707, Korea

Tel: +82-2-950-1114, Fax: +82-2-950-1039

E-mail: $22890 @$ paik.ac.kr or neck and right shoulder pain with progressive right side weakness while falling asleep. During consult, she had reported taking anti-hypertensive medication for 20 years and was followed regularly for hepatitis C. She reported no trauma history or physical exertion for the past few weeks. Neurological examination resulted in alert mental status and the detection of right hemiparesis. Motor power of her right upper limb was grade III and ipsilateral hand grasping power was grade II. In her right lower extremity, motor power was grade IV, and her left whole extremities were intact. Right side hypoesthesia was also observed. Based on her medical history and clinical symptoms, the physician working at the emergency department ordered brain CT to rule out intracranial hemorrhage. However, brain CT did not show any positive findings (Fig. 1-A). Subsequent brain diffuse MRI was taken to rule out cerebral infarction, but it was also negative (Fig. 1-B, C). Cervical MRI was performed to rule out a cervical lesion based on the patient's report of posterior neck pain and revealed a mass like lesion in the right posterior epidural
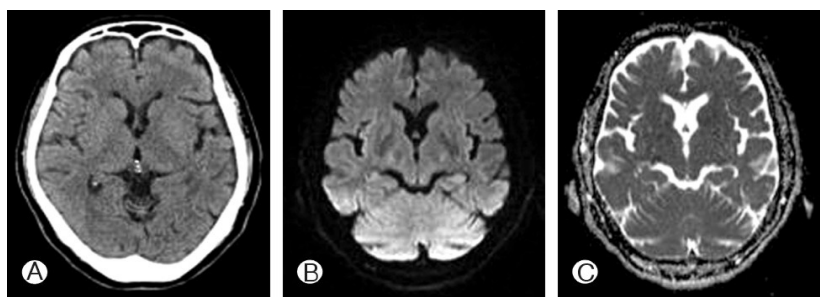

Fig. 1. Brain $C T(A)$ and $M R$ diffusion images $(B, C)$ showing no intracranial lesion. 

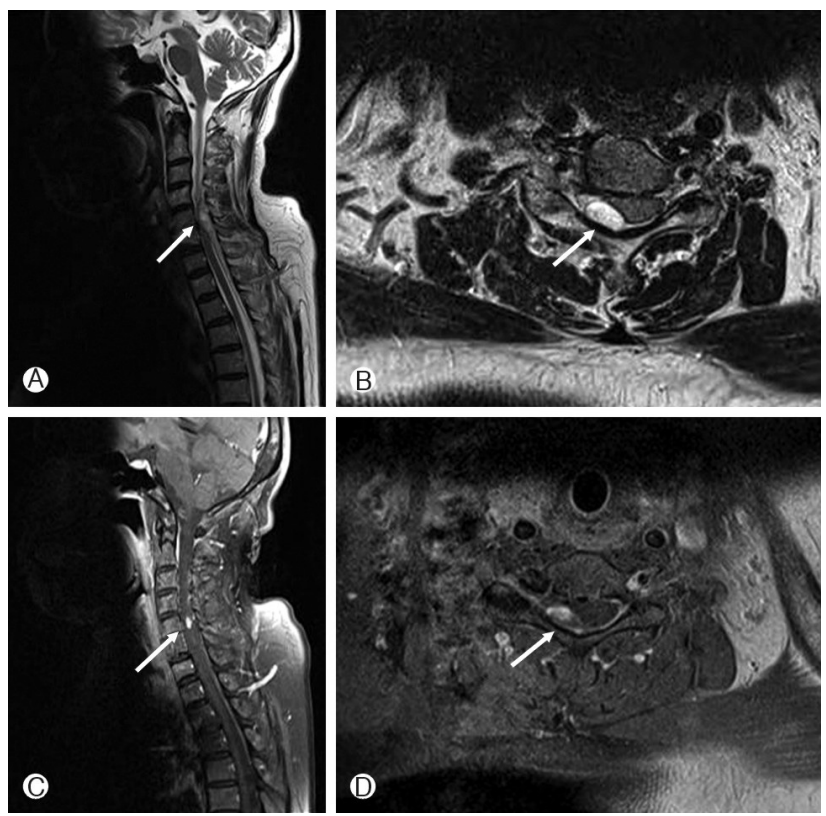

Fig. 2. Mid sagittal $(A, C)$ and axial MRI $(B, D)$ of the cervical spine showing a mass like lesion with enhancement.

space at the C3-6 level compressing the right side of the spinal cord. The mass was isointensive to the spinal cord on T1WI and heterogeneously hyperintensive with central high signal foci on T2WI. At the C5 level, the MRI showed an ovoid, enhancing, nodular lesion, which was a suspected tumorous condition such as hemangioma or hemangiopericytoma (Fig. 2). Initial laboratory tests showed only severe thrombocytopenia $\left(45,000 / \mathrm{mm}^{3}\right)$. As her neurologic signs were getting worse with time, we decided to perform surgical intervention. Under general endotracheal anesthesia via light wand intubation, right C5 unilateral total laminectomy and C6 partial hemilaminectomy were performed. The mass lesion was pure hematoma and was removed (Fig. 3). We could find neither vascular malformation or an active bleeding point. She went into rehabilitation after her operation and finally achieved complete neurologic recovery and relief of neck and shoulder pain.

\section{DISCUSSION}

Spontaneous spinal epidural hematoma is a rare disease and a total of 470 cases have been reported worldwide since $1869^{9}$. Beatty et al. described SCEDH as idiopathic spontaneous spinal epidural hematoma ${ }^{4}$. Lonjon et al. used "nontraumatic spinal epidural hematoma" to describe idiopathic SCEDH with liver cirrhosis secondary to some other pathology ${ }^{13)}$. Because various descriptions have been used for idiopathic spinal EDH, we used "spontaneous" to emphasize "the absence of any trauma or iatrogenic injury". Risk factors of SCEDH
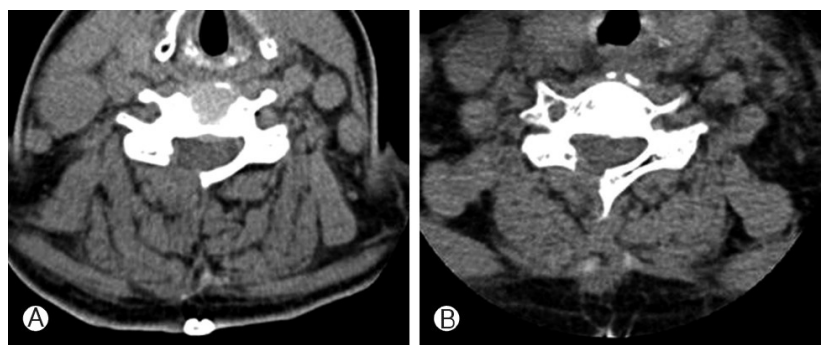

Fig. 3. Cervical axial $C T$ images after surgery showing complete hematoma evacuation (A: C5 level, B: C6 level, respectively).

include hypertension, coagulopathy, anti-coagulant use, Paget's disease, tumor genesis, and vascular malformations. However, an exact bleeding source and mechanism of hematoma have not yet been revealed.

It is generally accepted that most cervical epidural hematomas arise from rupture of the epidural venous plexus ${ }^{3,8,10)}$. However, it is still being debated whether the origin of bleeding in acute SCEDH is arterial or venous. Some authors have favored an arterial origin as the bleeding source. For example, Beatty and Winston postulated that the source of bleeding was the 'free' anastomotic arteries in the epidural space that connect with radicular arteries ${ }^{4}$. The probability of arterial bleeding is also supported by the rapid development of spinal cord compression ${ }^{16}$. Conversely, Bruyn and Bosma theorized that spontaneous spinal epidural hematoma occurs due to local pooling within valve-less, thin-walled epidural veins and brief increases in intravenous pressure (caused by intra-thoracic and intra-abdominal pressure elevations) leading to epidural vein rupture $^{5}$. This theory of how venous pooling and pressure causes vessel vulnerability could explain why bleeding occurs in SCEDH with liver cirrhosis, and possibly accounts for the reported associations with voiding, bending, turning in bed at night, straining while toileting, sneezing, and coitus ${ }^{5,8)}$. Liver cirrhosis is a systemic disease that affects the entire circulatory system and causes abnormalities in venous plexuses in the cervical region. In addition to coagulation abnormalities, liver cirrhosis causes portal vein hypertension, resulting in the development of collateral venous flow from splanchnic circulation. The epidural veins, like the azygous or hemiazygous thoracic veins, would be swollen and their walls would become thin. Thus, in patients with liver cirrhosis, the epidural venous plexus can easily be damaged with neurologic deficits becoming more readily apparent with less blood pooling in epidural spaces. However, our case did not show any evidence of definite venous plexus or vascular malformation, grossly.

The clinical manifestations of SCEDH can resemble those of a ruptured cervical disc, an epidural mass, or a dissecting aortic aneurysm ${ }^{15}$. Typical clinical features are sudden, localized, intensive pain around the involved vertebrae, often show 
radiculopathy which mimic disc rupture. Some authors insisted that the characteristics clinical symptoms of SCEDH are acute onset of radicular paresthesia and localized pain and progressive paraplegia and loss of sensory function within minutes or hours ${ }^{15)}$. However, it is not always easy to diagnose SCEDH because various symptoms may are often confused with myelitis, polyradiculitis, myelocompressive disease including tumor, intracranial lesions such as aneurysmal rupture, or thalamic hemorrhage. SCEDH may sometimes present as hemiparesis or hemiplegia without neck pain. Hsieh et al. reported a case of SCEDH in a 65 -year-old man complaining of acute onset unilateral hemiparesis the initial presentation ${ }^{12}$. Patients with hemiparesis due to SCEDH have often been misdiagnosed as having cerebral infarction and had been treated with anti-platelet therapy of anti-coagulants ${ }^{1,12,19)}$. The symptoms of the present case were enough to lead to misdiagnosis even though the epidural hematoma compressed the cervical cord and neural foramen directly.

MRI is the most useful method for positive diagnosis of spontaneous and secondary cervical epidural hematoma. Many authors reported various signal intensities with differing time intervals. Matsumura et al. reported that SCEDH usually exhibits an isointense signal to the spinal cord within $24 \mathrm{~h}$ after symptom onset and a hyperintense signal after 36 hours on T1 $\mathrm{WI}^{14)}$. Groen et al. reported a characteristic hyperintensity with hypointense foci on $\mathrm{T} 2 \mathrm{WI}^{8)}$. Melanie et al. insisted that SCEDH showed various intensities including hyper-, iso- or hypointensity on T1WI and did not correlate with time from symptom onset to imaging. They also reported that it showed heterogeneously hyperintense to spinal cord with foci of hypointensity on $\mathrm{T}_{2} \mathrm{WI}^{7}$. However, MRI findings in the present case showed a different pattern compared to previous reports. It showed isointensity to spinal cord on T1WI, but heterogeneous hyperintensity with central foci of hyperintensity on T2WI that did change with time. Nodular enhancing ovoid lesion was suspected with tumorous condition such as hemangiopericytoma or hemangioma.

Several cases of SCEDH have been reported to treat conservatively ${ }^{2,11,18)}$. All cases involved mild neurological symptoms and quadriplegia at examination. However, many neurosurgeons still advocate rapid diagnosis and surgical hematoma as the standard management to recover neurologic deterioration. Choi et al. emphasize rapid surgical decompression in cases of symptomatic epidural hematoma ${ }^{6}$. Groen et al. reported significantly better outcomes for patients with complete neurologic deficit that underwent decompression within $36 \mathrm{~h}$ of symptom onset and within $48 \mathrm{~h}$ in patients with incomplete deficit ${ }^{8}$. Shin et al. emphasized the importance of early decompression at less than $12 \mathrm{~h}$ for incomplete neurological deficits associated with ischemic change, and recommended a shorter time to surgical intervention to promote positive neurological outcomes $^{17)}$. In our case, surgery was performed within $24 \mathrm{~h}$ of the development of initial neurologic signs. Although our patient had bleeding tendency due to severe thrombocytopenia, her postoperative state was favorable with no evidence of complication. Our experiences and the literature suggest that recovery of neurological impairment is related to the time elapsed between symptom onset and surgical decompression. Accurate and early diagnosis of SCEDH and prompt surgical intervention, within $24 \mathrm{~h}$, alleviates sensory motor deficits and may contribute to complete neurologic recovery ${ }^{17}$.

\section{CONCLUSION}

Spontaneous cervical epidural hematoma is a rare disorder and can present various clinical patterns and easily be misdiagnosed. As was shown by our case, early diagnosis based on MRI findings and hematoma evacuation within $24 \mathrm{~h}$ of symptom onset can lead to full neurologic recovery in patients with spontaneous cervical epidural hematoma.

\section{REFERENCES}

1. Adamson DC, Bulsara K, Bronec PR: Spontaneous cervical epidural hematoma: case report and literature review. Surg Neurol 62:156-159; discussion 159-160, 2004

2. Anderson TJ, Donaldson IM: Spontaneous resolution of cervical spinal epidural haematoma. Postgrad Med J 65:488-490, 1989

3. Awada A, Russell N, al Fayez N, Naufal R, al Kohlani H: Spontaneous cervical epidural hematoma: case report. Spinal Cord 36:71-72, 1998

4. Beatty RM, Winston KR: Spontaneous cervical epidural hematoma. A consideration of etiology. J Neurosurg 61:143-148, 1984

5. Bruyn GW BN: Spinal extradural hematoma. Handbook of Clinical Neurology. Vol 26. Amsterdam; North-Holland Publishing Co., 1976

6. Choi JH, Kim JS, Lee SH: Cervical spinal epidural hematoma following cervical posterior laminoforaminotomy. J Korean Neurosurg Soc 53:125-128, 2013

7. Fukui MB, Swarnkar AS, Williams RL: Acute spontaneous spinal epidural hematomas. AJNR Am J Neuroradiol 20:1365-1372, 1999

8. Groen RJ, Ponssen H: The spontaneous spinal epidural hematoma. A study of the etiology. J Neurol Sci 98:121-138, 1990

9. Grollmus J, Hoff J: Spontaneous spinal epidural haemorrhage: good results after early treatment. J Neurol Neurosurg Psychiatry 38:89-90, 1975

10. Gundry CR, Heithoff KB: Epidural hematoma of the lumbar spine: 18 surgically confirmed cases. Radiology 187:427-431, 1993

11. Halim TA, Nigam V, Tandon V, Chhabra HS: Spontaneous cervical epidural hematoma: report of a case managed conservatively. Indian J Orthop 42:357-359, 2008 
12. Hsieh CF, Lin HJ, Chen KT, Foo NP, et al: Acute spontaneous cervical spinal epidural hematoma with hemiparesis as the initial presentation. Eur J Emerg Med 13:36-38, 2006

13. Lonjon MM, Paquis P, Chanalet S, Grellier P: Nontraumatic spinal epidural hematoma: report of four cases and review of the literature. Neurosurgery 41:483-486; discussion 486-487, 1997

14. Matsumura A, Namikawa T, Hashimoto R, Okamoto T, Yanagida I, Hoshi $\mathrm{M}$, et al: Clinical management for spontaneous spinal epidural hematoma: diagnosis and treatment. Spine J 8: 534-537, 2008

15. Pear BL: Spinal epidural hematoma. Am J Roentgenol Radium
Ther Nucl Med 115:155-164, 1972

16. Scott BB, Quisling RG, Miller CA, Kindt GW: Spinal epidural hematoma. JAMA 235:513-515, 1976

17. Shin JJ, Kuh SU, Cho YE: Surgical management of spontaneous spinal epidural hematoma. Eur Spine J 15:998-1004, 2006

18. Vaya A, Resureccion M, Ricart JM, Ortuno C, Ripoll F, Mira Y, et al: Spontaneous cervical epidural hematoma associated with oral anticoagulant therapy. Clin Appl Thromb Hemost 7: 166-168, 2001

19. Wang CC, Chang CH, Lin HJ, Lin KC, Kuo JR: Misdiagnosis of spontaneous cervical epidural haemorrhage. Eur Spine J 18 Suppl 2:210-212, 2009 